\title{
MINIMIZATION OF THE DISCRETIZATION ERROR IN MASS AND STIFFNESS FORMULATIONS BY AN INVERSE METHOD
}

\author{
H. AHMADIAN, ${ }^{1}$ M. I. FRISWELL ${ }^{2}$ AND J. E. MOTTERSHEAD ${ }^{1 *}$ \\ ${ }^{1}$ Department of Mechanical Engineering, The University of Liverpool, U.K. \\ ${ }^{2}$ Department of Mechanical Engineering, The University of Wales, Swansea, U.K.
}

\begin{abstract}
This paper is concerned with the formulation of mass and stiffness matrices. In the direct approach one uses assumed shape functions to develop the mass and stiffness terms. Alternatively, we may construct the matrices by using an inverse approach; the terms are assigned so that the difference between an analytical model and a numerical (discrete) one is minimized. Here we show that more accurate models can be obtained by the latter approach. The accuracy of rod, beam and plate elements that have been developed by both of the approaches are discussed, and an accurate model of a rectangular plate is obtained by using the inverse method. The superior performance of the new element compared to other established models is demonstrated for the cases of static and dynamic response of a clamped plate. (C) 1998 John Wiley \& Sons, Ltd.
\end{abstract}

Int. J. Numer. Meth. Engng., 41, 371-387 (1998)

KEY WORDS: plate elements; inverse methods; discretisation; static and dynamic analysis

\section{INTRODUCTION}

In the development of element mass and stiffness matrices for vibration analysis, one starts with an assumed displacement solution for the element based on nodal variables, and the mass and stiffness coefficients are then determined by the minimization of either an energy functional or the residues of the equation of motion. Different assumed solutions, or shape functions, lead to different mass and stiffness models. However, provided that the shape functions satisfy certain requirements, the solutions of assembled models representing the same system, but obtained by using different shape functions, converge to the same result as the number of elements increase. This means that the choice between different allowable shape functions mainly affects the rate of convergence of the solution.

Considerable effort in finite element modelling is focused on obtaining an element formulation that gives a small discretization error and fast convergence. The accuracy of the finite element solution is usually assessed via a posteriori error estimators. Two major types of a posteriori error

*Correspondence to: J. E. Mottershead, Department of Mechanical Engineering, University of Liverpool, PO Box 147, Liverpool L69 3BX, U.K.

Contract grant sponsor: European Union BRITE EURAM

CCC 0029-5981/98/020371-17\$17.50

Received 8 January 1996

(C) 1998 John Wiley \& Sons, Ltd.

Revised 17 September 1996 
estimators have been developed: the residual type of error estimator presented by Babuska and Rheinboldt ${ }^{1}$ and the post-processing type of error estimator proposed by Zienkiewicz and Zhu. ${ }^{2}$ The accuracy of the solution and the error estimate is improved by implementing a sequence of refinements to the mesh. It is of interest in this paper that the error analysis may be embedded in element formulations by an inverse approach, instead of being used as an a posteriori test. The inverse method was used by Argyris et al., ${ }^{3}$ Bergan et al., ${ }^{4}$ and Simo and Rafai ${ }^{5}$ to enforce constraints, in the form of assumed strain modes, on the stiffness formulation to guarantee that the element model would pass the patch test. However, the requirement to pass the patch test is only a necessary condition, and is not sufficient in itself to ensure that the formulation will be representative of the physics.

The error in the solution of a finite element model can be expressed by a series in powers of $\Delta x$, where $\Delta x$ is the element characteristic length. Apparently, it was Stavrinidis ${ }^{6}$ and his co-workers who first used this type of analysis for assessing the discretization error in rod and beam elements. The coefficients of this series are a linear combination of the terms in the mass and stiffness matrices. The objective in this paper is to define the terms in element mass and stiffness matrices by minimizing the error in the finite element solution.

In general, an element model must meet certain requirements. Consider an element with $d$ degrees of freedom and $r$ rigid-body modes, $\phi_{i}, i=1, \ldots, r$. The mass matrix $\mathbf{M}$ is symmetric, positive-definite and of rank $d$, whilst the stiffness matrix $\mathbf{K}$ is symmetric and positive-semidefinite. The rigid-body modes of the element, $\boldsymbol{\Phi}_{\mathrm{R}}=\left[\phi_{1}, \phi_{2}, \ldots, \phi_{r}\right]$, form the null space of the stiffness matrix such that

$$
\mathbf{K} \Phi_{\mathrm{R}}=0
$$

If the rigid-body modes are defined on the principal co-ordinates of the element then

$$
\boldsymbol{\Phi}_{\mathrm{R}}^{\mathrm{T}} \mathbf{M} \boldsymbol{\Phi}_{\mathrm{R}}=\operatorname{diag}\left(m, m, m, I_{x x}, I_{y y}, I_{z z}\right)
$$

where $m$ is the element mass and $I_{x x}, I_{y y}$ and $I_{z z}$ are the moments of inertia. Moreover, if the element has some symmetric properties, then the mass and stiffness models reflect these properties; rotation of the element about its symmetry axes does not change the mass and stiffness matrices. It is possible to define a set of mass and stiffness matrices for an element which satisfies these requirements but depend upon one or more parameters. Established elements are members of this set. By using finite element error analysis we are able to establish the accuracy of a model that arises from specified shape functions with known parameters, and to find the optimum values of the parameters. The idea may be demonstrated for the simplest of structural entities such as rods and beams.

\section{PARAMETRIC MODELS AND ERROR ANALYSIS FOR RODS AND BEAMS}

We consider a uniform rod of length, $L$, density, $\rho$, cross-sectional area, $A$, and Young's modulus, $E$. Longitudinal displacement, $u(x, t)$, of the vibrating rod can be modelled by using a set of rod elements. Each element may be represented by a mass matrix, $\mathbf{M}$, and a stiffness matrix, $\mathbf{K}$, both of which are real, symmetric and of dimension 2 . The element has one rigid-body mode $\phi_{1}=[1,1]^{\mathrm{T}}$. Then, by applying the symmetry considerations and constraints defined in equations (1) and (2), 
we obtain the following parametric model:

$$
\begin{gathered}
\mathbf{K}=k\left[\begin{array}{rr}
1 & -1 \\
-1 & 1
\end{array}\right] \quad k>0 \\
\mathbf{M}=\rho A \Delta x\left[\begin{array}{cc}
\frac{1}{2}-\theta & \theta \\
\theta & \frac{1}{2}-\theta
\end{array}\right], \quad \theta<1 / 4
\end{gathered}
$$

where $\Delta x$ is the length of the rod element and $k, \theta$ are two parameters with real values. When the rod is modelled by a string of identical rod elements that are connected in a straight line and defined in equations (3) and (4), then the equation of the $i$ th node in the assembled finite element model (i.e. the $i$ th row of the assembled matrices) gives that,

$$
k\left(-u_{i-1}+2 u_{i}-u_{i+1}\right)+\rho A \Delta x\left(\theta \ddot{u}_{i-1}+(1-2 \theta) \ddot{u}_{i}+\theta \ddot{u}_{i+1}\right)=0, \quad i=2, \ldots, n
$$

where $n=L / \Delta x$ is the number of elements. We want to set the parameters $k$ and $\theta$ so that equation (5) represents the governing equation for free vibrations of a rod,

$$
E \frac{\partial^{2} u}{\partial x^{2}}-\rho \ddot{u}=0
$$

By using a Taylor series expansion for $u_{i-1}$ and $u_{i+1}$ we may rewrite equation (5) as

$$
\left(k \Delta x \frac{\partial^{2} u_{i}}{\partial x^{2}}-\rho A \ddot{u}_{i}\right)+\sum_{m=1}^{\infty} \frac{2 \Delta x^{2 m}}{(2 m) !}\left(\frac{k \Delta x \quad \partial^{2(m+1)} u_{i}}{(2 m+1)(2 m+2) \partial x^{2(m+1)}}-\rho A \theta \frac{\partial^{2 m} \ddot{u}_{i}}{\partial x^{2 m}}\right)=0
$$

When $k=E A / \Delta x$ the first term in equation (7) represents the governing equation for free vibration of a uniform rod and the residual represents the finite element formulation error. Equation (7) converges to equation (6) if and only if $\Delta x \rightarrow 0$. We must now determine the arbitrary parameter $\theta$. Different well-established models may be associated with particular values of $\theta$. For example, $\theta=\frac{1}{6}$ produces a consistent mass matrix based on the assumed shape functions $N_{1}=x / \Delta x, \quad N_{2}=1-x / \Delta x ; \theta=\frac{1}{8}$ results in a mass matrix based on shape functions $N_{1}=\cos (\pi x / 2 \Delta x)^{2}, N_{2}=\sin (\pi x / 2 \Delta x)^{2}$; and a lumped-mass model is obtained when $\theta=0$. It appears from equation (7) that the discretization error of these models is of the order $\Delta x^{2}$.

Another approach to obtain $\theta$ is to minimize the difference between equations (6) and (7). This is achieved when $\theta=\frac{1}{12}$. In this case the second-order terms of equation (7) also reproduce equation (6) and the discretization error is now of the fourth order. The error analysis shows that between members of the family of models defined in (5) the one specified with $k=E A / \Delta x$, and $\theta=\frac{1}{12}$ represents the longitudinal vibration of the rod best. $\mathrm{MacNeal}^{7}$ obtained the same result by minimizing the error in the eigenvalue estimates of a rod. The shape functions for $\theta=\frac{1}{12}$ are obscure but the model can be determined by averaging the consistent and lumped-mass models.

This simple example shows that by using the error analysis, we are able not only to assess the performance of different models of an element obtained using various shape functions, but we may also find the best possible model for the element. Shape functions related to the model with optimum choice of parameters may or may not be known to us but this is of little concern because the best model has been found. For stress analysis (where derivatives of the solution are required) one may use an interpolation scheme to define a displacement field with appropriate continuity over a group or all of the elements. 
In a similar manner, one may develop a family of acceptable models for a uniform beam element. The result is a stiffness matrix with two parameters and a mass matrix with four parameters,

$$
\begin{gathered}
\mathbf{K}=k\left[\begin{array}{rrrc}
1 & \frac{1}{2} & -1 & \frac{1}{2} \\
& \alpha & -\frac{1}{2} & \frac{1}{2}-\alpha \\
& & 1 & -\frac{1}{2} \\
\text { Sym. } & & \alpha
\end{array}\right] \\
\mathbf{M}=\rho A \Delta x\left[\begin{array}{llcc}
m_{1,1} & m_{1,2} & \frac{1}{2}-m_{1,1} & m_{1,4} \\
& m_{2,2} & -m_{1,4} & m_{2,4} \\
& & m_{1,1} & -m_{1,2} \\
\text { Sym. } & & & m_{2,2}
\end{array}\right] \\
m_{2,4}=\frac{1}{6}-m_{1,1 / 2}+m_{1,2}+m_{1,4}-m_{2,2}
\end{gathered}
$$

The choice of

$$
\begin{gathered}
k=12 \frac{E I}{(1+g) \Delta x^{3}} \\
\alpha=(4+g) / 12 \\
m_{1,1}=\left(\frac{13}{35}+\frac{7}{10} g+\frac{1}{3} g^{2}\right) /(1+g)^{2} \\
m_{1,2}=\left(\frac{11}{210}+\frac{11}{120} g+\frac{1}{24} g^{2}\right) /(1+g)^{2} \\
m_{1,4}=-\left(\frac{13}{420}+\frac{3}{40} g+\frac{1}{24} g^{2}\right) /(1+g)^{2} \\
m_{2,2}=\left(\frac{1}{105}+\frac{1}{60} g+\frac{1}{120} g^{2}\right) /(1+g)^{2}
\end{gathered}
$$

produces the consistent Timoshenki beam element, ${ }^{8}$ where EI is the rigidity of the beam and $g$ is the ratio between shear and bending effects. The consistent Euler-Bernoulli beam with Hermitian cubic-shape functions is obtained when $g=0$.

In order to find the best element formulation for a beam, we may assemble the parametric model and compare it with the governing equation of the beam. In comparing the parametric model with the governing equation of the Euler-Bernoulli beam, we find the optimum values for stiffness parameters are $k=12 E I / \Delta x^{3}, \alpha=\frac{1}{3}$, which is consistent with the classical formulation. However, the resultant mass matrix is different from any of the established ones. It is generally known that the accuracy in vibration analysis of a beam can be improved if one uses a mass matrix which is different from the established lumped or consistent mass matrices. The results obtained for the rod mass matrix might lead one to consider the weighted averaging of consistent and lumped-mass matrices for beams, examples of which can be found in the works of Park and Jensen $^{9}$ and Kim. ${ }^{10}$ Stavrinidis et al. ${ }^{6}$ assumed that the beam element stiffness matrix was correct and by using the discretization error showed that the optimum values for the mass matrix terms 
were:

$$
m_{1,1}=\frac{163}{420}, \quad m_{1,2}=\frac{51}{840}, \quad m_{1,4}=-\frac{19}{840}, \quad m_{2,2}=\frac{15}{840}
$$

The resultant mass matrix leads to an accuracy of fourth order in vibration analysis which cannot be obtained by a linear combination of the consistent and lumped models.

The stiffness matrices for rod and beam elements obtained by using the conventional displacement formulation and the inverse approach are the same and the shape functions can be uniquely defined by a complete polynomial. The advange of using an inverse approach over the conventional one can be appreciated when the displacement of an element cannot be expressed using a complete polynomial. A clear example of this is the Kirchoff plate element. Here we use an inverse approach to obtain an accurate model for both stiffness and mass matrices of a plate element. There are a bewildering array of plate elements: triangular, rectangular, quadrilateral; Kirchoff or Mindlin-Reissner; three or more degrees-of-freedom per node. We consider the family of 12 degree-of-freedom rectangular Kirchoff plate elements and produce a parametric model. The number of independent parameters is determined by the necessary conditions on the model to comply with the Kirchoff plate theory. An error analysis can be carried out which shows that the existing rectangular-plate element models (i.e. particular values of the independent parameters) have discretization errors of order 4. We obtain the optimum parameters for a rectangular-plate element so that it has the minimum discretization error. The performance of the new formulation is demonstrated by comparing the predictions of the optimal model to those from existing models and the exact solution.

\section{PARAMETRIC PLATE MODEL}

We consider a rectangular-plate element with four nodes (one at each corner) and three degrees-of-freedom at each node. The displacement vector for such an element can be written as

$$
\begin{gathered}
\mathbf{d}=\left[\mathbf{d}_{1}, \mathbf{d}_{2}, \mathbf{d}_{3}, \mathbf{d}_{4}\right]^{\mathrm{T}} \\
\mathbf{d}_{i}=\left[w_{i}, \Delta y \alpha_{i}, \Delta x \beta_{i}\right]^{\mathrm{T}}, \quad i=1, \ldots, 4
\end{gathered}
$$

where $w_{i}, \alpha_{i}$ and $\beta_{i}$ represent the deflection and rotations at the $i$ th node and $\Delta x$ and $\Delta y$ are the dimensions of the element as shown in Figure 1 . The element has a $12 \times 12$ symmetric,

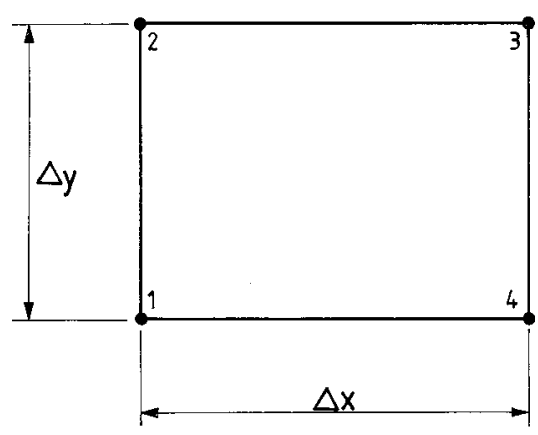

Figure 1. Plate element with four nodes 
positive-semi-definite stiffness matrix that can be written in the general form

$$
\mathbf{K}=k\left[\begin{array}{llll}
\mathbf{K}_{11} & \mathbf{K}_{12} & \mathbf{K}_{13} & \mathbf{K}_{14} \\
& \mathbf{K}_{22} & \mathbf{K}_{23} & \mathbf{K}_{24} \\
& & \mathbf{K}_{33} & \mathbf{K}_{34} \\
\text { Sym. } & & & \mathbf{K}_{44}
\end{array}\right]
$$

where $k$ is a positive real scalar. $\mathbf{K}_{i j}, i, j=1, \ldots, 4$, are $3 \times 3$ submatrices with non-dimensional entries, and $\mathbf{K}_{i i}=\mathbf{K}_{i i}^{\mathrm{T}}$.

The element has two symmetry axes as shown in Figure 2, and when the element is rotated through $\pi$ radians about one of these axes the stiffness matrix remains unchanged. The rotations are equivalent to applying the transformations

$$
\begin{aligned}
\mathbf{T}_{x x} & =\left[\begin{array}{llll}
0 & 0 & 0 & \mathbf{R} \\
0 & 0 & \mathbf{R} & 0 \\
0 & \mathbf{R} & 0 & 0 \\
\mathbf{R} & 0 & 0 & 0
\end{array}\right] \\
\mathbf{T}_{y y} & =\left[\begin{array}{llll}
0 & \mathbf{S} & 0 & 0 \\
\mathbf{S} & 0 & 0 & 0 \\
0 & 0 & 0 & \mathbf{S} \\
0 & 0 & \mathbf{S} & 0
\end{array}\right]
\end{aligned}
$$

where

$$
\begin{aligned}
& \mathbf{R}=\operatorname{diag}(-1,-1,1) \\
& \mathbf{S}=\operatorname{diag}(-1,1,-1)
\end{aligned}
$$

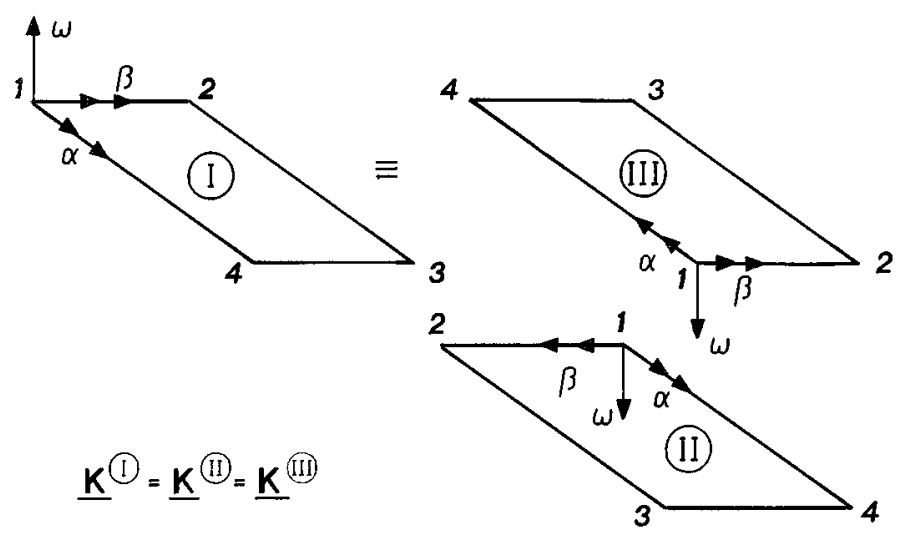

Figure 2. Symmetry of the plate 
A comparison of the transformed stiffness matrices to the original stiffness (in equation (23)) reveals that

$$
\mathbf{K}=k\left[\begin{array}{cccc}
\mathbf{K}_{11} & \mathbf{K}_{12} & \mathbf{K}_{13} & \mathbf{K}_{14} \\
& \mathbf{S K}_{11} \mathbf{S} & \mathbf{S K}_{14} \mathbf{S} & \mathbf{S K}_{13} \mathbf{S} \\
& & \mathbf{S R K}_{11} \mathbf{R S} & \mathbf{S R K}_{12} \mathbf{R S} \\
\text { Sym. } & & & \mathbf{R K}_{11} \mathbf{R}
\end{array}\right]
$$

where

$$
\begin{aligned}
\mathbf{K}_{11} & =\mathbf{K}_{11}^{\mathrm{T}} \\
\mathbf{K}_{12} & =\mathbf{S K}_{12}^{\mathrm{T}} \mathbf{S} \\
\mathbf{K}_{13} & =\mathbf{S} \mathbf{R} \mathbf{K}_{13}^{\mathrm{T}} \mathbf{R S} \\
\mathbf{K}_{14} & =\mathbf{R K}_{14}^{\mathrm{T}} \mathbf{R}
\end{aligned}
$$

It is apparent that the stiffness matrix given by equation (28) is unaffected by either of the transformations in equations (24) and (25). The constraint of physical symmetry reduces the number of unattributed stiffness parameters to 24 : the stiffness matrix is defined by a $3 \times 3$ symmetric matrix and three other $3 \times 3$ matrices, which apart from a sign change, are also symmetric.

When the element is rotated through $\pi / 2$ radians in its own plane then the ratio of side dimensions, given by $p=\Delta y / \Delta x$, is inverted. ${ }^{\dagger}$ This rotation is equivalent to the transformation

$$
\begin{aligned}
\mathbf{T}_{z z} & =\left[\begin{array}{llll}
0 & \mathbf{Q} & 0 & 0 \\
0 & 0 & \mathbf{Q} & 0 \\
0 & 0 & 0 & \mathbf{Q} \\
\mathbf{Q} & 0 & 0 & 0
\end{array}\right] \\
\mathbf{Q} & =\left[\begin{array}{rrr}
1 & 0 & 0 \\
0 & 0 & -1 \\
0 & 1 & 0
\end{array}\right]
\end{aligned}
$$

from which it is found that

$$
\begin{aligned}
& \mathbf{K}_{11}(1 / p)=\mathbf{Q}^{\mathrm{T}} \mathbf{R} \mathbf{K}_{11}(p) \mathbf{R} \mathbf{Q} \\
& \mathbf{K}_{13}(1 / p)=\mathbf{Q}^{\mathrm{T}} \mathbf{R} \mathbf{K}_{13}(p) \mathbf{R} \mathbf{Q} \\
& \mathbf{K}_{14}(1 / p)=\mathbf{Q}^{\mathrm{T}} \mathbf{R} \mathbf{K}_{12}(p) \mathbf{R} \mathbf{Q}
\end{aligned}
$$

It leads directly from equations (35)-(37) that if $k_{1,2}, k_{1,4}, k_{1,5}, k_{1,6}, k_{1,8}, k_{2,2}, k_{2,5}, k_{2,6}, k_{2,8}$ and $k_{3,6}$ are known then $-k_{1,3}, k_{1,10},-k_{1,12},-k_{1,11},-k_{1,9}, k_{3,3}, k_{3,12},-k_{2,12}, k_{3,9}$ and $k_{2,11}$, respectively, are given by changing $p$ to $1 / p$. This produces 10 more constraints so that the

${ }^{\dagger}$ For the case of an orthotropic material the flexural rigidity along each direction, $D_{x}$ and $D_{y}$, are also interchanged 
number of unattributed parameters is reduced to 14 . We find that $k_{1,1}, k_{2,3}, k_{1,7}$ and $k_{2,9}$ remain unchanged by the rotation defined in equations (33) and (34).

The mass matrix, $\mathbf{M}$, is found by the same approach as above to have the same structure as the stiffness matrix

$$
\mathbf{M}=\rho \Delta x \Delta y\left[\begin{array}{cccc}
\mathbf{M}_{11} & \mathbf{M}_{12} & \mathbf{M}_{13} & \mathbf{M}_{14} \\
& \mathbf{S M}_{11} \mathbf{S} & \mathbf{S M}_{14} \mathbf{S} & \mathbf{S M}_{13} \mathbf{S} \\
& & \mathbf{S R M}_{11} \mathbf{R S} & \mathbf{S R M}_{12} \mathbf{R S} \\
\text { Sym. } & & & \mathbf{R M}_{11} \mathbf{R}
\end{array}\right]
$$

where

$$
\begin{aligned}
& \mathbf{M}_{11}=\mathbf{M}_{11}^{\mathrm{T}} \\
& \mathbf{M}_{12}=\mathbf{S M}_{12}^{\mathrm{T}} \mathbf{S} \\
& \mathbf{M}_{13}=\mathbf{S} \mathbf{R} \mathbf{M}_{13}^{\mathrm{T}} \mathbf{R S} \\
& \mathbf{M}_{14}=\mathbf{R} \mathbf{M}_{14}^{\mathrm{T}} \mathbf{R}
\end{aligned}
$$

and $\rho$ is the mass density per unit area of the plate. The relationship of the mass terms $m_{1,2}, m_{1,4}$, $m_{1,5}, m_{1,6}, m_{1,8}, m_{2,2}, m_{2,5}, m_{2,6}, m_{2,8}, m_{3,6}$, with the terms $m_{1,3}, m_{1,10}, m_{1,12}, m_{1,11}, m_{1,9}, m_{3,3}$, $m_{3,12}, m_{2,12}, m_{3,9}, m_{2,11}$ is the same as in the corresponding stiffness terms.

The element has three rigid-body modes, one representation of which is

$$
\begin{aligned}
& \boldsymbol{\Phi}_{R}=\left[\begin{array}{c}
\text { SAS } \\
\mathbf{A} \\
\text { RAR } \\
\text { SRARS }
\end{array}\right] \\
& \mathbf{A}=\left[\begin{array}{lll}
1 & \frac{1}{2} & \frac{1}{2} \\
0 & 1 & 0 \\
0 & 0 & 1
\end{array}\right]
\end{aligned}
$$

The rigid-body modes must occupy the null space of $\mathbf{K}$, as expressed in equation (1), and thus

$$
\mathbf{K}_{11} \mathrm{SAS}+\mathrm{K}_{12} \mathrm{~A}+\mathrm{K}_{13} \mathrm{RAR}+\mathrm{K}_{14} \mathrm{SRARS}=0
$$

This leads to nine equations of which five are independent.

The mass constraints expressed in equation (2) result in three equations to determine the entries of the mass matrix,

$$
\begin{gathered}
m_{1,1}+m_{1,4}+m_{1,7}+m_{1,10}=\frac{1}{4} \\
m_{1,1}-m_{1,4}-m_{1,7}+m_{1,10} \\
+4\left(m_{2,2}-m_{1,2}+m_{2,5}-m_{1,5}+m_{2,8}-m_{1,8}+m_{2,11}-m_{1,11}=\frac{1}{12}\right.
\end{gathered}
$$




$$
\begin{aligned}
& m_{1,1}+m_{1,4}-m_{1,7}-m_{1,10} \\
& \quad+4\left(m_{3,3}+m_{1,3}+m_{3,12}+m_{1,12}+m_{3,9}+m_{1,9}+m_{3,6}+m_{1,6}=\frac{1}{12}\right.
\end{aligned}
$$

Two of the equations (46)-(48) are independent.

The stiffness matrix of the plate element with nine independent parameters and the mass matrix with 12 independent parameters can be formed by using the physical constraints described above. ${ }^{\ddagger}$ To fix these remaining stiffness and mass terms we can convert the discrete finite element equations to differential equations and compare them to the governing equations from classical plate theory. The parametric plate element formed on the basis of the physical constraints above can represent either the Kirchoff plate theory or the Reissner-Mindlin theory. In what follows, we consider the construction of a Kirchoff plate element by the inverse approach.

\section{ERROR ANALYSIS FOR THE KIRCHOFF PLATE ELEMENT}

We assemble the elements with area $A=\Delta x \times \Delta y$ to give a regular mesh for a rectangular plate with free edges. Figure 3 shows four internal elements connected at a common node $(i, j)$ where three finite element equations can be written in the form

$$
\begin{aligned}
\mathbf{K}_{31} & \mathbf{d}_{i-1, j-1}+\left(\mathbf{K}_{32}+\mathbf{K}_{41}\right) \mathbf{d}_{i-1, j}+\mathbf{K}_{42} \mathbf{d}_{i-1, j+1} \\
& +\left(\mathbf{K}_{34}+\mathbf{K}_{21}\right) \mathbf{d}_{i, j-1}+\left(\mathbf{K}_{11}+\mathbf{K}_{22}+\mathbf{K}_{33}+\mathbf{K}_{44}\right) \mathbf{d}_{i, j} \\
& +\left(\mathbf{K}_{43}+\mathbf{K}_{12}\right) \mathbf{d}_{i, j-1}+\mathbf{K}_{24} \mathbf{d}_{i+1, j-1}+\left(\mathbf{K}_{23}+\mathbf{K}_{14}\right) \mathbf{d}_{i+1, j} \\
& +\mathbf{K}_{13} \mathbf{d}_{i+1, j+1}+\mathbf{M}_{31} \ddot{\mathbf{d}}_{i-1, j-1}+\left(\mathbf{M}_{32}+\mathbf{M}_{41}\right) \ddot{\mathbf{d}}_{i-1, j} \\
& +\mathbf{M}_{42} \ddot{\mathbf{d}}_{i-1, j+1}+\left(\mathbf{M}_{34}+\mathbf{M}_{21}\right) \ddot{\mathbf{d}}_{i, j-1} \\
& +\left(\mathbf{M}_{11}+\mathbf{M}_{22}+\mathbf{M}_{33}+\mathbf{M}_{44}\right) \ddot{\mathbf{d}}_{i, j}+\left(\mathbf{M}_{43}+\mathbf{M}_{12}\right) \ddot{\mathbf{d}}_{i, j+1} \\
& +\mathbf{M}_{24} \ddot{\mathbf{d}}_{i+1, j-1}+\left(\mathbf{M}_{23}+\mathbf{M}_{24}\right) \ddot{\mathbf{d}}_{i+1, j}+\mathbf{M}_{13} \ddot{\mathbf{d}}_{i+1, j+1}=\mathbf{0}
\end{aligned}
$$

The displacements and rotations at node $i+1, j+1$ can be written in terms of the displacement and rotations, and their derivatives with respect to $x$ and $y$, at node $i, j$ by using the Taylor series expansion

$$
\mathbf{d}_{i+1, j+1}=\mathbf{d}_{i, j}+\sum_{n=1}^{\infty} \frac{1}{n !}\left(\Delta x \frac{\partial}{\partial x}+\Delta y \frac{\partial}{\partial y}\right)^{n} \mathbf{d}_{i, j}
$$

Similar expressions can be written to refer the displacements and rotations at nodes $i-1, j+1$; $i-1, j ; i-1, j-1 ; i, j+1 ; i, j-1 ; i+1, j ; i+1, j-1$ to the displacement and rotations, and

\footnotetext{
${ }^{\ddagger}$ The stiffness and mass parameters are a function of two geometrical variables $p=\Delta y / \Delta x$ and the area $A=\Delta x \times \Delta y$. Other plate elements have two or more geometrical variables. For example, the geometry of a triangular-plate element can be uniquely defined using three geometrical variables: its area and two of its angles. Using the inverse approach one determines the relationship between these geometrical variables and the terms in the element stiffness and mass matrices for the type of element (rectangular, triangular, quadrilateral, etc.) under study. In the case of the rectangular-plate element, physical symmetry of the element reduces the number of unknowns and simplifies the analysis. In other elements where the physical symmetry does not exist, more terms in the model must be identified from the error analysis
} 


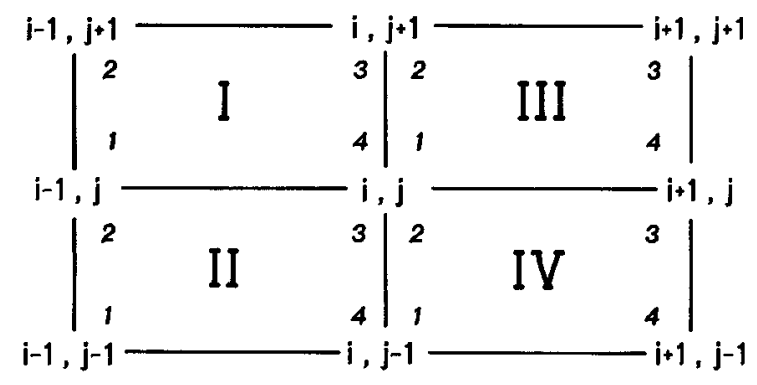

Figure 3. Assembled plate structure

their derivatives at node $i, j \S$ The accelerations and angular accelerations can be treated in the same way. This process has the effect of transforming the discrete finite element equation (49) into three differential equations having terms of increasing order of smallness, $O\left(\Delta x^{2 n}, \Delta y^{2 n}\right)$, $n=0,1,2, \ldots$. The terms of order zero may be compared with the governing equation from classical plate theory. It is found that the zero-order terms introduce no information on the stiffness and mass parameters that has not already been found from the analysis of the physical constraints. Next, we try comparing the second-order terms with the classical plate equation, and if the differential equation is satisfied at that order of smallness we proceed to investigate the fourth-order terms, and so on. When an order of smallness is reached so that the unattributed stiffness and mass parameters are exhausted and the differential equation cannot be reconciled with the classical equation then the order of the discretization error has been determined.

The equations that result from the second-order terms in the Taylor series are

$$
\begin{aligned}
\frac{A^{2} k}{6}\left[\left(k_{1,10}+k_{1,7}-4 k_{1,12}-4 k_{1,9}\right) / p^{2} \frac{\partial^{4} w}{\partial x^{4}}+6\left(k_{1,7}+2 k_{1,8}-2 k_{1,9}\right) \frac{\partial^{4} w}{\partial x^{2} \partial y^{2}}\right. & \\
+ & \left.p^{2}\left(k_{1,4}+k_{1,7}+4 k_{1,5}+4 k_{1,8}\right) \frac{\partial^{4} w}{\partial y^{4}}\right]+\rho A \ddot{w}=0 \\
2 A^{2} k \frac{\partial}{\partial x} & {\left[\left(k_{3,12}+k_{3,9}+k_{1,12} / 3+k_{1,9} / 3\right) / p^{2} \frac{\partial^{2} w}{\partial x^{2}}+\left(k_{3,6}+k_{3,9}-2 k_{2,9}+k_{1,9}\right) \frac{\partial^{2} w}{\partial y^{2}}\right]=0 } \\
2 A^{2} k \frac{\partial}{\partial y} & {\left[\left(k_{2,11}+k_{2,8}-k_{1,8}-2 k_{2,9}\right) \frac{\partial^{2} w}{\partial x^{2}}+p^{2}\left(k_{2,5}+k_{2,8}-k_{1,5} / 3-k_{1,8} / 3\right) \frac{\partial^{2} w}{\partial y^{2}}\right]=0 }
\end{aligned}
$$

By comparing equation (51) with the Kirchoff plate equation,

$$
D\left(\frac{\partial^{4} w}{\partial x^{4}}+2 \frac{\partial^{4} w}{\partial x^{2} \partial y^{2}}+\frac{\partial^{4} w}{\partial y^{4}}\right)+\rho \ddot{w}=0
$$

\footnotetext{
${ }^{\S}$ The same procedure can be applied straightforwardly for other element types such as triangular, quadrilateral, etc.
} 
where $D=E t^{3} / 12\left(1-v^{2}\right)$ is the flexural rigidity of the plate, and bearing in mind that in general $\partial^{2} w / \partial x^{2} \neq 0, \partial^{2} w / \partial y^{2} \neq 0$, we find that $k=D / A$ and

$$
\begin{aligned}
k_{1,10}+k_{1,7}-4 k_{1,12}-4 k_{1,9} & =6 p^{2} \\
k_{1,7}+2 k_{1,8}-2 k_{1,9} & =2 \\
k_{1,4}+k_{1,7}+4 k_{1,5}+4 k_{1,8} & =6 / p^{2} \\
k_{3,12}+k_{3,9}+k_{1,12} / 3+k_{1,9} / 3 & =0 \\
k_{3,6}+k_{3,9}-2 k_{2,9}+k_{1,9} & =0 \\
k_{2,11}+k_{2,8}-k_{1,8}-2 k_{2,9} & =0 \\
k_{2,5}+k_{2,8}-k_{1,5} / 3-k_{1,8} / 3 & =0
\end{aligned}
$$

These introduce four more independent constraints on the entries of K. Further restrictions can be obtained by considering the terms at the boundaries of the plate. There are three equations relating to a node on a free edge normal to the $x$-direction of which their lowest order terms after simplification are

$$
\begin{gathered}
D p \Delta x \frac{\partial}{\partial x}\left[\frac{\partial^{2} w}{\partial x^{2}}+\left(k_{1,7}-k_{1,6}+2 k_{1,8}-k_{1,9}\right) \frac{\partial^{2} w}{\partial y^{2}}\right]=0 \\
2 A \Delta y\left(k_{28}+k_{2,11}-k_{1,8}-k_{2,9}-k_{2,6}\right) \frac{\partial^{2} w}{\partial x \partial y}=0 \\
D p \Delta x\left[-\frac{\partial^{2} w}{\partial x^{2}}+\left(2 k_{2,6}-2 k_{2,9}-k_{1,6}+k_{1,9}\right) \frac{\partial^{2} w}{\partial y^{2}}\right]=0
\end{gathered}
$$

Comparing equations (62) and (64) with the boundary conditions of the plate,

$$
\begin{gathered}
\frac{\partial}{\partial x}\left[\frac{\partial^{2} w}{\partial x^{2}}+(2-v) \frac{\partial^{2} w}{\partial y^{2}}\right]=0 \\
{\left[\frac{\partial^{2} w}{\partial x^{2}}+v \frac{\partial^{2} w}{\partial y^{2}}\right]=0}
\end{gathered}
$$

we obtain

$$
\begin{gathered}
\left(k_{1,7}-k_{1,6}+2 k_{1,8}-k_{1,9}\right)=2-v \\
\left(2 k_{2,6}-2 k_{2,9}-k_{1,6}+k_{1,9}\right)=-v
\end{gathered}
$$

Equation (67) ensures that at the edge of the plate $\partial^{2} \omega / \partial x \partial y=0$ and satisfies equation (63). This brings the total number of constraints introduced by the Kirchoff plate theory to six and, hence, reduces the number of parameters of stiffness to only three parameters. We take $k_{1,5}, k_{2,8}$ and $k_{2,9}$ as the independent parameters of the stiffness matrix.

All the stiffness models for the rectangular Kirchoff plate element reported in the literature belong to the family of models formed via the parametric stiffness matrix. Different models may be associated with different values of $k_{1,5}, k_{2,8}$, and $k_{2,9} \cdot k_{1,5}=2 / p^{2}+(1-v) / 5$, 
$k_{2,8}=1 / 3 p^{2}+(1-v) / 15, k_{2,9}=0$ give the stiffness matrix of a non-conforming element reported by Melosh, ${ }^{11}$ and Zienkiewicz and Chung, ${ }^{12}$ the MZC model. The stiffness of the conforming element developed by Bogner et al., ${ }^{13}$ the BFS model, is obtained when $k_{1,15}=-13 p^{2} / 35+78 / 35 p^{2}+6 / 25, \quad k_{2,8}=3 p^{2} / 35+9 / 35 p^{2}+2 / 25, \quad k_{2,9}=-13\left(p^{2}+1 / p^{2}\right) /$ $70+1 / 50$. Assigning $k_{1,5}=2 / p^{2}, k_{2,8}=1 / 3 p^{2}, k_{2,9}=0$, results in a stiffness matrix obtained by Przemieniecki ${ }^{14}$ by the flexibility method. To evaluate the discretization error in these models and to see if there is any possibility to improve the plate model we consider the fourth-order terms in internal nodes, from which we obtain

$$
\begin{aligned}
& \frac{4 D A^{2}}{6 !}\left[12 / p \frac{\partial^{6} w}{\partial x^{6}}+30 c_{1} / p \frac{\partial^{6} w}{\partial x^{4} \partial y^{2}}+30 c_{2} p \frac{\partial^{6} w}{\partial x^{2} \partial y^{4}}+12 p \frac{\partial^{6} w}{\partial y^{6}}\right] \\
& +2 \rho A^{2}\left[\left(m_{1,10}+m_{1,7}-2 m_{1,12}-2 m_{1,9}\right) / p \frac{\partial^{2}}{\partial x^{2}}+p\left(m_{1,4}+m_{1,7}+2 m_{1,5}+2 m_{1,8}\right) \frac{\partial^{2}}{\partial y^{2}}\right] \ddot{w} \\
& =0 \\
& \quad \frac{D A^{2}}{3 p} \frac{\partial}{\partial x}\left[\frac{1}{5} \frac{\partial^{4} w}{\partial x^{4}}+\left(3 k_{3,9}-2 k_{2,9}-k_{1,12}-3 p^{2}\right) \frac{\partial^{4} w}{\partial x^{2} \partial y^{2}}-p^{2} k_{2,9} \frac{\partial^{4} w}{\partial y^{4}}\right] \\
& \quad+4 \rho A^{2}\left(m_{3,3}+m_{3,6}+m_{1,9}+m_{3,9}+m_{1,12}+m_{3,12}\right) / p \frac{\partial \ddot{w}}{\partial x}=0 \\
& \quad \frac{D A^{2} p}{3} \frac{\partial}{\partial y}\left[-k_{2,9} / p^{2} \frac{\partial^{4} w}{\partial x^{4}}+\left(3 k_{2,8}-2 k_{2,9}+k_{1,5}-3 / p^{2}\right) \frac{\partial^{4} w}{\partial x^{2} \partial y^{2}}+\frac{1}{5} \frac{\partial^{4} w}{\partial y^{4}}\right] \\
& \quad+4 \rho A^{2} p\left(m_{2,2}+m_{2,5}+m_{2,8}+m_{2,11}-m_{1,5}-m_{1,8}\right) \frac{\partial \ddot{w}}{\partial y}=0
\end{aligned}
$$

where $c_{1}=k_{1,12}+3 p^{2}+1$, and $c_{2}=-k_{1,5}+3 / p^{2}+1$. Equation (69) represents the Kirchoff plate theory if and only if we set $k_{15}=\left(13 / p^{2}+1\right) / 5$ and restrict the entries of $\mathbf{M}$ as

$$
\begin{gathered}
m_{1,10}+m_{1,7}-2 m_{1,12}-2 m_{1,9}=4 / 5 ! \\
m_{1,4}+m_{1,7}+2 m_{1,5}+2 m_{1,8}=4 / 5 !
\end{gathered}
$$

This converts the first of the fourth-order terms to

$$
\frac{8}{5 !} A^{2}\left(\frac{1}{p} \frac{\partial^{2}}{\partial x^{2}}+p \frac{\partial^{2}}{\partial y^{2}}\right)\left[D\left(\frac{\partial^{4} w}{\partial x^{4}}+2 \frac{\partial^{4} w}{\partial x^{2} \partial y^{2}}+\frac{\partial^{4} w}{\partial y^{4}}\right)+\rho \ddot{w}\right]=0
$$

Necessary conditions for (70) to represent the governing equation of the plate is $k_{2,9}=-1 / 5 p^{2}$, while (71) requires $k_{2,9}=-p^{2} / 5$. Minimizing the error in these equations, we find $k_{2,9}=-\left(p^{2}+\right.$ $\left.1 / p^{2}\right) / 10, k_{2,8}=\left(1+1 / p^{2}-p^{2}\right) / 15$, and subsequently obtain

$$
\begin{gathered}
m_{3,3}+m_{3,6}+m_{1,9}+m_{3,9}+m_{1,12}+m_{3,12}=\frac{1}{60} \\
m_{2,2}+m_{2,5}+m_{2,8}+m_{2,11}-m_{1,5}-m_{1,8}=\frac{1}{60}
\end{gathered}
$$


Using the above, the last two fourth-order terms are simplified to

$$
\begin{aligned}
& \frac{1}{15} A^{2} \frac{\partial}{\partial x}\left[D\left(\frac{\partial^{4} w}{\partial x^{4}}+2 \frac{\partial^{4} w}{\partial x^{2} \partial y^{2}}+\frac{1}{2}\left(p^{4}+1\right) \frac{\partial^{4} w}{\partial y^{4}}\right)+\rho \ddot{w}\right]=0 \\
& \frac{1}{15} A^{2} \frac{\partial}{\partial y}\left[D\left(\frac{1}{2}\left(1 / p^{4}+1\right) \frac{\partial^{4} w}{\partial x^{4}}+2 \frac{\partial^{4} w}{\partial x^{2} \partial y^{2}}+\frac{\partial^{4} w}{\partial y^{4}}\right)+\rho \ddot{w}\right]=0
\end{aligned}
$$

These terms deviate from the Kirchoff plate theory when $p \neq 1$. We specified the optimum stiffness parameters, and using equations (72), (73), (75) and (76) introduced more constraints on the mass matrix terms. Both MZC and BFS mass matrices satisfy equations (72), (73), (75) and (76); however, their stiffness matrices, along with Przemieniecki's stiffness matrix are less than optimal. The established rectangular models for the Kirchoff plate have discretization errors of order 4.

When $k_{1,5}=\left(13 / p^{2}+1\right) / 5, k_{2,9}=-\left(p^{2}+1 / p^{2}\right) / 10$, and $k_{2,8}=\left(1+1 / p^{2}-p^{2}\right) / 15$ the result is a more accurate stiffness matrix, with the submatrices $\mathbf{K}_{11}, \mathbf{K}_{12}, \mathbf{K}_{13}$ and $\mathbf{K}_{14}$ given by

$$
\begin{aligned}
& \mathbf{K}_{11}=\left[\begin{array}{ccc}
2\left(7+13\left(p^{2}+1 / p^{2}\right) / 5\right. & \left(13 / p^{2}+1\right) / 5+v & -\left(13 p^{2}+1\right) / 5-v \\
& 2\left(2+p^{2}+14 / p^{2}\right) / 15 & \left(p^{2}+1 / p^{2}\right) / 10-v \\
\text { Sym. } & & 2\left(2+1 / p^{2}+14 p^{2}\right) / 15
\end{array}\right] \\
& \mathbf{K}_{12}=\left[\begin{array}{ccc}
2\left(2 p^{2}-7-13 / p^{2}\right) / 5 & \left(13 / p^{2}+1\right) / 5 & \left(1-2 p^{2}\right) / 5+v \\
-\left(13 / p^{2}+1\right) / 5 & \left(p^{2}+14 / p^{2}-1\right) / 15 & -\left(p^{2}+1 / p^{2}\right) / 10 \\
\left(1-2 p^{2}\right) / 5+v & \left(p^{2}+1 / p^{2}\right) / 10 & 2\left(p^{2}-1 / p^{2}-2\right) / 15
\end{array}\right] \\
& \mathbf{K}_{13}=\left[\begin{array}{ccc}
2\left(7-2\left(p^{2}+1 / p^{2}\right)\right) / 5 & \left(2 / p^{2}-1\right) / 5 & -\left(2 p^{2}-1\right) / 5 \\
-\left(2 / p^{2}-1\right) / 5 & \left(1+1 / p^{2}-p^{2}\right) / 15 & -\left(p^{2}+1 / p^{2}\right) / 10 \\
\left(2 p^{2}-1\right) / 5 & -\left(p^{2}+1 / p^{2}\right) / 10 & \left(1+p^{2}-1 / p^{2}\right) / 15
\end{array}\right] \\
& \mathbf{K}_{14}=\left[\begin{array}{ccc}
2\left(2 / p^{2}-7-13 p^{2}\right) / 5 & \left(2 / p^{2}-1\right) / 5-v & -\left(13 p^{2}+1\right) / 5 \\
\left(2 / p^{2}-1\right) / 5-v & 2\left(1 / p^{2}-p^{2}-2\right) / 15 & \left(p^{2}+1 / p^{2}\right) / 10 \\
\left(13 p^{2}+1\right) / 5 & -\left(p^{2}+1 / p^{2}\right) / 10 & \left(1 / p^{2}+14 p^{2}-1\right) / 15
\end{array}\right]
\end{aligned}
$$

When $p=1$ the stiffness matrix introduced in equations (79)-(82) has a fourth-order accuracy and, hence, the discretization error is of order 6 at the internal nodes. The mass matrix can be determined by minimizing the error either in the internal nodes or in the nodes located on a free edge. In both cases the requirements for $\mathbf{M}$ that were determined earlier must be retained. Considering the error at the edge of the plate, it is found that the second and fourth-order terms are automatically satisfied by the previously established relationships for the mass terms, but a fixed third-order error remains. We may satisfy the fifth-order terms and in that case the result will be a mass matrix which is very similar to the MZC element. Instead, we minimise the error in 
the internal nodes so that the submatrices $\mathbf{M}_{11}, \mathbf{M}_{12}, \mathbf{M}_{13}$ and $\mathbf{M}_{14}$ take the values of

$$
\begin{aligned}
& \mathbf{M}_{11}=\frac{1}{2721600}\left[\begin{array}{lll}
408402 & 54432 & -54432 \\
& 14058 & -9372 \\
\text { Sym. } & 14058
\end{array}\right] \\
& \mathbf{M}_{12}=\frac{1}{2721600}\left[\begin{array}{ccc}
119718 & -25353 & -28188 \\
25353 & -6147 & -6248 \\
-28188 & 6248 & 6462
\end{array}\right] \\
& \mathbf{M}_{13}=\frac{1}{2721600}\left[\begin{array}{ccc}
32562 & -5427 & 5427 \\
5427 & 207 & 3024 \\
-5427 & 3024 & 207
\end{array}\right] \\
& \mathbf{M}_{14}=\frac{1}{2721600}\left[\begin{array}{rrr}
119718 & 28188 & 25353 \\
28188 & 6462 & 6248 \\
-25353 & -6248 & -6147
\end{array}\right]
\end{aligned}
$$

The proposed mass matrix provides the highest possible accuracy at the internal nodes whilst producing the same order of error as the MZC element on a free edge. In what follows, we consider the improvement that the new model provides in predicting the plate behaviour.

\section{NUMERICAL EXAMPLE}

A test problem was used to determine the efficiency of the new plate element. Static and dynamic behaviour of a fully clamped square plate was predicted using the new formulation and compared with that obtained from established shape-function formulations and the analytical solution. Other researchers have used the same problem as a test case for plate elements. ${ }^{15-18}$ From the existing models, we only list the results of MZC formulation; the stiffness parameters $k_{1,5}, k_{2,8}$, and $k_{2,9}$ in the MZC formulation are the closest to the optimum values and, hence, produce smaller errors when compared to other models reported in the literature. ${ }^{15}$

Table I. Dimensionless centre displacement, $w D / q L^{4}$ for uniform load $q$

\begin{tabular}{lccccc}
\hline & \multicolumn{2}{c}{ MZC model } & & \multicolumn{2}{c}{ Inverse model } \\
\cline { 2 - 3 } \cline { 5 - 6 } Mesh & $p=1$ & $p=2$ & & $p=1$ & $p=2$ \\
\hline $4 \times 4$ & $0 \cdot 001403$ & $0 \cdot 002778$ & & $0 \cdot 001274$ & $0 \cdot 002561$ \\
$8 \times 8$ & $0 \cdot 001304$ & $0 \cdot 002593$ & & $0 \cdot 001266$ & $0 \cdot 002540$ \\
$12 \times 12$ & $0 \cdot 001283$ & 0.002560 & & $0 \cdot 001265$ & $0 \cdot 002536$ \\
$16 \times 16$ & $0 \cdot 001275$ & $0 \cdot 002548$ & & $0 \cdot 001265$ & $0 \cdot 002535$ \\
Exact $^{19}$ & $0 \cdot 001265$ & $0 \cdot 002533$ & & $0 \cdot 001265$ & $0 \cdot 002533$ \\
\hline
\end{tabular}



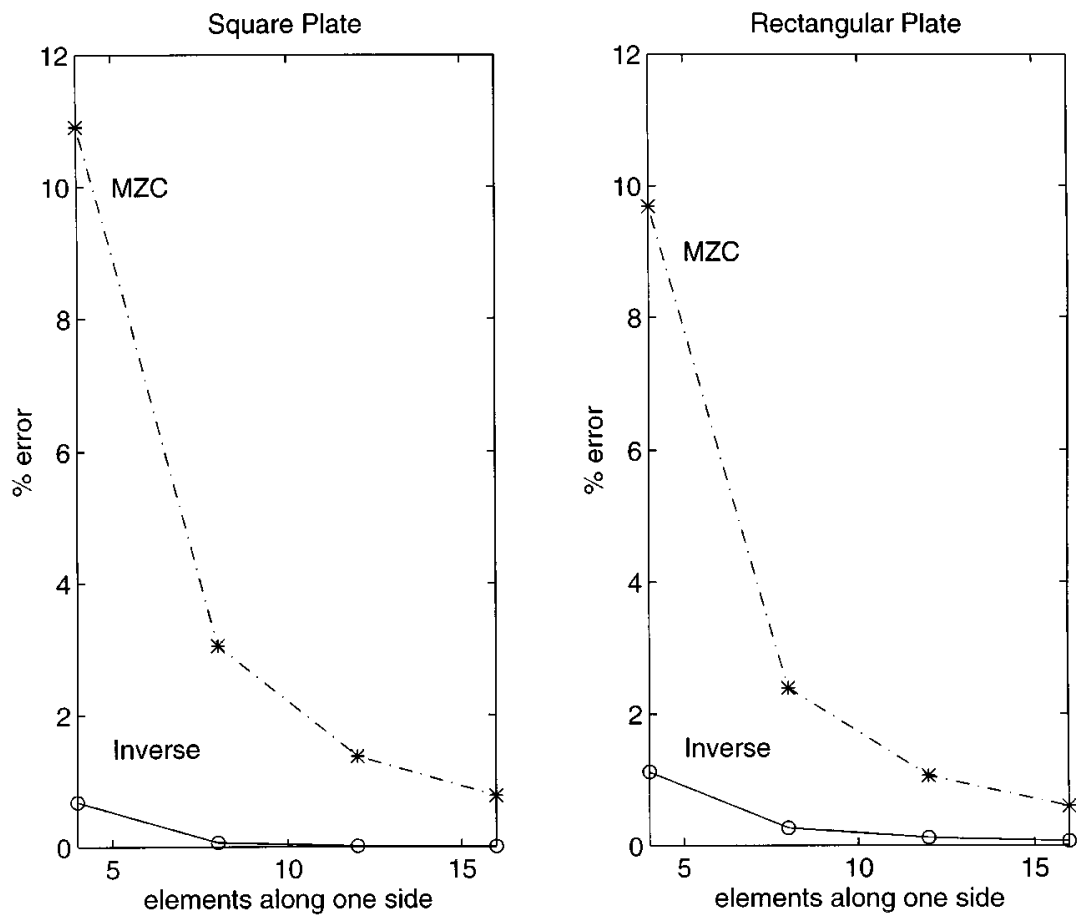

Figure 4. Errors in the estimated centre displacement of a clamped plate

Table II. Dimensionless natural frequency of a square fully clamped plate $\omega L^{2} \sqrt{(\rho / D)}$

\begin{tabular}{|c|c|c|c|c|c|c|c|c|}
\hline \multirow{2}{*}{$\begin{array}{l}\text { Rayleigh- } \\
\text { Ritz }^{20}\end{array}$} & \multicolumn{4}{|c|}{ MZC model } & \multicolumn{4}{|c|}{ Inverse model } \\
\hline & $4 \times 4$ & $8 \times 8$ & $12 \times 12$ & $16 \times 16$ & $4 \times 4$ & $8 \times 8$ & $12 \times 12$ & $16 \times 16$ \\
\hline 35.98 & $34 \cdot 31$ & $35 \cdot 45$ & $35 \cdot 74$ & $35 \cdot 84$ & $35 \cdot 87$ & 35.97 & $35 \cdot 98$ & $35 \cdot 98$ \\
\hline $73 \cdot 39$ & 70.03 & $72 \cdot 04$ & $72 \cdot 74$ & $73 \cdot 01$ & $73 \cdot 18$ & $73 \cdot 36$ & $73 \cdot 39$ & $73 \cdot 39$ \\
\hline $73 \cdot 39$ & $70 \cdot 03$ & $72 \cdot 04$ & $72 \cdot 74$ & $73 \cdot 01$ & $73 \cdot 18$ & $73 \cdot 36$ & $73 \cdot 39$ & $73 \cdot 39$ \\
\hline $108 \cdot 22$ & $98 \cdot 06$ & $103 \cdot 71$ & $106 \cdot 00$ & $106 \cdot 92$ & $108 \cdot 02$ & $108 \cdot 06$ & $108 \cdot 18$ & $108 \cdot 20$ \\
\hline $131 \cdot 58$ & $127 \cdot 58$ & $129 \cdot 41$ & $130 \cdot 44$ & $130 \cdot 90$ & $129 \cdot 39$ & $131 \cdot 52$ & $131 \cdot 57$ & $131 \cdot 58$ \\
\hline $132 \cdot 20$ & $129 \cdot 62$ & $130 \cdot 28$ & $131 \cdot 16$ & $131 \cdot 58$ & $130 \cdot 47$ & $132 \cdot 13$ & $132 \cdot 19$ & $132 \cdot 20$ \\
\hline $165 \cdot 00$ & $151 \cdot 01$ & $156 \cdot 95$ & $160 \cdot 83$ & $162 \cdot 52$ & $164 \cdot 55$ & $164 \cdot 71$ & $164 \cdot 92$ & $164 \cdot 97$ \\
\hline $165 \cdot 00$ & $151 \cdot 01$ & $156 \cdot 95$ & $160 \cdot 83$ & $162 \cdot 52$ & $164 \cdot 55$ & $164 \cdot 71$ & $164 \cdot 92$ & $164 \cdot 97$ \\
\hline
\end{tabular}

Table I shows the computed central deflection of a square fully clamped plate with area $L^{2}$ and a rectangular fully clamped plate with the area $L \times H, H / L=2$, both under uniformly distributed loads. Comparing the values obtained using the MZC and the new formulations, we notice a significant improvement in predicting the static behaviour of the plate using the new formulation. The errors in predicting the central deflection from both methods are shown in Figure 4. 

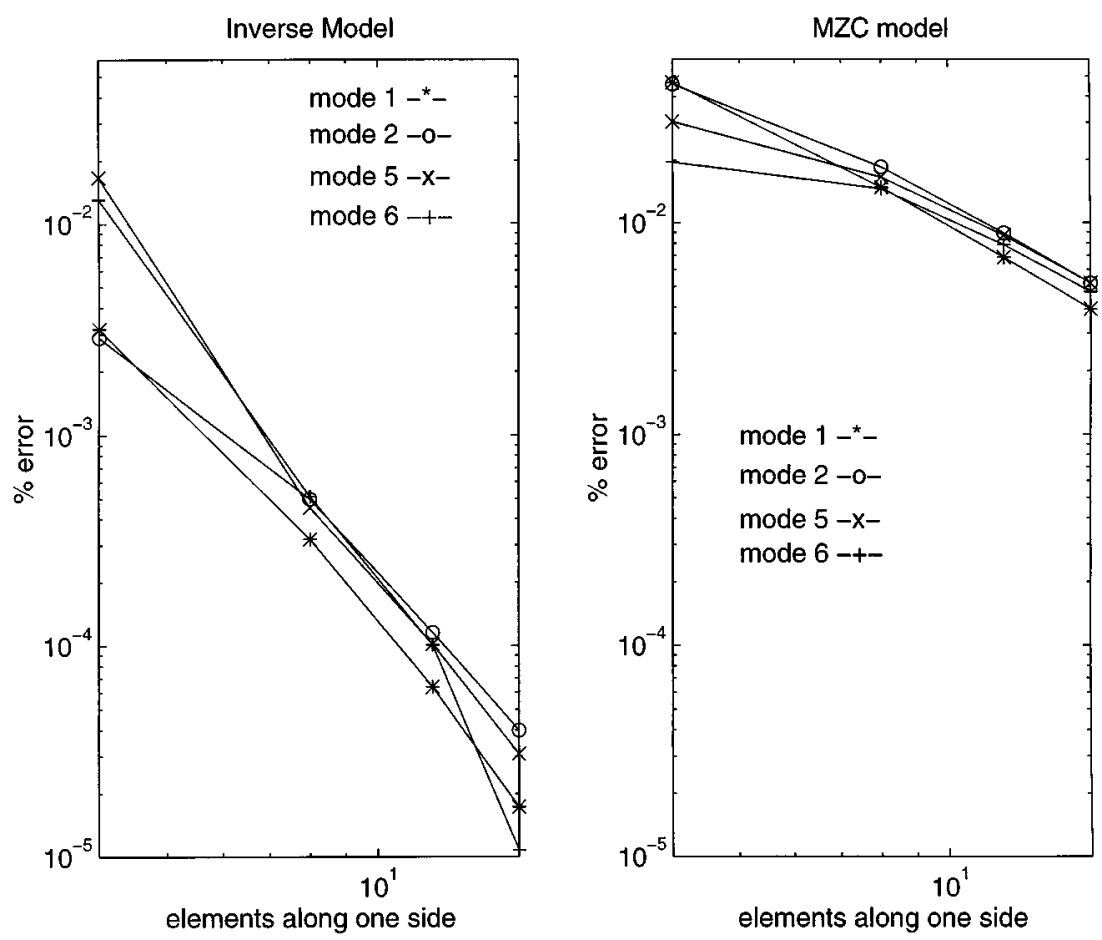

Figure 5. Errors in the estimated eigenvalues of a clamped square plate

Next we evaluate the eigenvalues of a square fully clamped plate obtained from the new model and from MZC model. The results for various meshes are reported in Table II. Also listed in this table are the eigenvalue solutions of the plate obtained by using the Rayleigh-Ritz method ${ }^{20}$ as the reference. Comparing the eigenvalue predictions we find that the results of the new formulation converge much faster than those obtained from the MZC model. Accuracy of the new model is of order 4 while in the MZC model the accuracy is of order 2. Therefore, the rate of convergence in the new model on $\log -\log$ scale is about twice the rate in the MZC model. This can be easily seen from Figure 5 which presents the percentage of error in eigenvalue estimates vs. number of elements along one side of the plate. Results from both examples shows the accuracy of the new plate model and its superiority over the established models in the literature.

\section{CONCLUSIONS}

A rather different approach from those usually employed in the finite element method is used to obtain the mass and stiffness matrices for a plate element. In the inverse approach, we consider what criteria must be satisfied by the element model and form a parametric family of admissible mass and stiffness matrices. The parameters of the element model are then obtained by minimising the discretization error in the element formulation. The benefit of using this approach is that it gives the best possible model for the element under consideration, whereas in the direct method the result is sensitive to the choice of shape functions and the optimum model is not achieved. 
An accurate plate element model is obtained by minimising the discretization error in formulation of mass and stiffness matrices. The improvements of static and dynamic responses are presented using a fully clamped rectangular plate example. The added accuracy in the new model requires no extra computational effort and it may be implemented easily into existing finite element codes.

ACKNOWLEDGEMENTS

This study was supported by the European Union BRITE EURAM scheme and forms part of the research conducted under project UPDYN.

\section{REFERENCES}

1. Babuska and W. C. Rheinboldt, 'Adaptive approaches and reliability estimates in finite element analysis', Comput. Meth. Appl. Mech. Engng., 17/18, 519-540 (1979).

2. O. C. Zienkiewicz and J. Z. Zhu, 'A simple error estimator and adaptive procedure for practical engineering analysis', Int. J. Numer. Meth. Engng., 24, 337-357 (1987).

3. J. H. Argyis, M. Haase and H.-P. Mlejnek, 'On an unconventional, but natural formulation of a stiffness matrix', Comput. Meth. Appl. Mech. Engng., 22, 1-22 (1980).

4. P. G. Bergan and M. K. Nygard, 'Finite elements with increased freedom in choosing shape functions', Int. J. Numer. Meth. Engng., 20, 643-663 (1984).

5. J. C. Simo and M. S. Rifai, 'A class of mixed assumed strain methods and the method of incompatible modes', Int. J. Numer. Meth. Engng., 29, 1595-1638 (1990).

6. C. Stavrinidis, J. Clinckemaillie and J. Dubois, 'New concepts for finite-element mass matrix formulations', $A I A A J$., 27(9), 1249-1255 (1989).

7. R. H. MacNeal, NASTRAN Theoretical Manual (level 16), NASA-sp-221, 1976.

8. Z. Friedman and B. Kosmatka, 'An improved two-noded Timoshenko beam finite element', Comput. Struct., 47(3), 473-481 (1993).

9. K. C. Park and D. D. Jensen, 'A systematic determination of Lumped and improved consistent mass matrices for vibration analysis', Proc. 30th Structural Dynamics and Materials Conf., Mobile, Alabama, U.S.A., April 1989, pp. $1532-1540$.

10. K. Kim, 'A review of mass matrices for eigenproblems', Comput. Struct., (6), 1041-1048 (1993).

11. R. J. Melosh, 'Basis for derivation of matrices for the direct stiffness method', AIAA J., 1(7), 1631-1637 (1963).

12. O. C. Zienkiewicz and Y. K. Cheung, 'The finite element method for the analysis of elastic isotropic and orthotropic slabs', Proc. Inst. Civil Engng., 28, 471-488 (1964).

13. F. K. Bogner, L. R. Fox and L. A. Schmit, 'The generation of interelement compatible stiffness and mass matrices by the use of interpolation formulas', Proc. Conf. Matrix Methods Structural Mechanics, AFIT, Wright-Patterson AF Base, OH, 1965, pp. 397-443.

14. J. S. Przemieniecki, Theory of Matrix Structural Analysis, McGraw-Hill, New York, 1968.

15. J. F. Abel and C. Desai, 'Comparison of finite elements for plate bending', J. Struct. Division Proc. ASCE, 98(ST9), 2143-2148 (1972).

16. T. J. R. Hughes, R. L. Taylor and W. Kanoknukulchai, 'A simple and efficient finite element for plate bending', Int. J. Numer. Meth. Engng., 11, 1529-1543 (1977).

17. J. L. Batoz, K. J. Bathe and L. W. Ho, 'A study of three-node triangular plate bending elements', Int. J. Numer. Meth. Engng., 15, 1771-1812 (1980).

18. B. Downs, 'Anticlastic curvature correction of a square plate finite element applied to the transverse vibration of flat plates', J. Sound Vib., 131(2), 249-258 (1989).

19. S. P. Timoshenko and S. Woinowsky-Krieger, Theory of Plates and Shells, McGraw-Hill, New York, 1959.

20. K. Vijayakumar and G. K. Ramaiah, 'Analysis of vibration of clamped square plates by Rayleigh-Ritz method with asymptotic solution from a modified Bolotin method', J. Sound Vib., 56, 127-135 (1978). 